\title{
Three-dimensional computed tomography mapping and analysis of distal femur fractures (AO/OTA types 33A, 33B, and 33C)
}

\author{
Ruiyang Li ${ }^{\#}$, Yingjie Zhuge ${ }^{\#}$, Yu Zhan^, Xuetao Xie^, Congfeng Luo^ \\ Department of Orthopaedic Surgery, Shanghai Jiao Tong University Affiliated Sixth People's Hospital, Shanghai, China \\ Contributions: (I) Conception and design: C Luo, R Li, Y Zhuge; (II) Administrative support: C Luo, X Xie; (III) Provision of study materials or \\ patients: R Li, Y Zhan; (IV) Collection and assembly of data: R Li, X Xie; (V) Data analysis and interpretation: R Li, Y Zhuge; (VI) Manuscript \\ writing: All authors; (VII) Final approval of manuscript: All authors. \\ \#These authors contributed equally to this work. \\ Correspondence to: Professor Congfeng Luo. Department of Orthopaedic Surgery, Shanghai Jiao Tong University Affiliated Sixth People's Hospital, \\ No. 600 Yishan Road, Shanghai 200233, China. Email: congfengl@outlook.com.
}

Background: Distal femur fractures are complex injuries with a high rate of fracture healing problems. Since the widespread of computed tomographic imaging in the diagnosis of distal femur fractures, many fracture characteristics have been discovered. This study aimed to depict the location and frequency of distal femur fracture lines and further analyze the morphological characteristics using the 3-dimensional computed tomography (CT) mapping technique, thus providing more information to solve this challenging clinical problem.

Methods: In total, 217 distal femur fractures in 216 patients were retrospectively reviewed. Fracture fragments on CT were digitally reconstructed and virtually reduced to match a template model. The contour of every fracture fragment was then marked with smooth curves, and the overlap of all fracture lines allowed for the creation of 3-dimensional fracture maps and heat maps. Fracture characteristics were summarized based on these maps.

Results: This study included 114 left knee injuries, 101 right knee injuries, and 1 case with bilateral injury. Distal femur fractures were most likely to occur among patients aged 61 to 70 years. On the heat map of all 217 fractures, fracture line hot zones were mainly concentrated around the metaphysis, the lateral part of the intercondylar notch, and the patellofemoral joint. Distal femur fractures with three Arbeitsgemeinschaft für Osteosynthesefragen Foundation/Orthopaedic Trauma Association (AO/OTA) types demonstrated distinct fracture characteristics. In total, there were 58 coronal plane fractures (41.1\%) in 141 intercondylar fractures. Conclusions: The intercondylar fracture patterns in AO/OTA type B and type C fractures were similar, while the supracondylar characteristics in AO/OTA type A and type $\mathrm{C}$ were different. The findings in this study can help orthopaedic surgeons better understand the fracture morphology on the basis of AO/OTA classification. Further studies are needed to establish a standard biomechanical fracture model and new fixation strategy for better clinical outcomes.

Keywords: Distal femur fracture; fracture morphology; 3-dimensional mapping (3D mapping); fracture classification

Submitted Sep 01, 2021. Accepted for publication Dec 31, 2021.

doi: $10.21037 / \mathrm{atm}-21-4591$

View this article at: https://dx.doi.org/10.21037/atm-21-4591

\footnotetext{
^ ORCID: Ruiyang Li, 0000-0003-4957-063X; Yingjie Zhuge, 0000-0003-2920-5037; Yu Zhan, 0000-0002-7551-5039; Xuetao Xie, 00000003-2159-0030; Congfeng Luo, 0000-0001-5876-5266.
} 


\section{Introduction}

Distal femur fractures are complex injuries that constitute $0.5 \%$ of all fractures (1) and $6 \%$ of femur fractures (2). These fractures are characterized by female predominance, and patient groups show a bimodal distribution, with highenergy trauma in younger patients and low-energy trauma in the elderly with osteoporosis $(2,3)$.

Since the incidence and proportion of the elderly in distal femur fractures are increasing due to the aging of the global population (3), this trend will likely result in a change of fracture morphology and complications. To date, studies available in the literature have mainly focused on implant fixation and postoperative complications. For distal femur fractures with comminuted metaphysis, fracture healing problems such as delayed union or nonunion, stiffness, hardware failure, and loss of alignment are not uncommon (4-7). However, a lack of research on fracture characteristics will be an obstacle to the development of operative fixation strategy and internal fixation design for these complex fractures. Due to these limitations, further research is needed to make a detailed description of the morphological characteristics of distal femur fractures, especially in a 3-dimensional manner.

Computed tomography (CT) has become an important tool in diagnosing and preoperative planning of complex distal femur fractures since Nork et al. (8) reported in 2005 that only $69 \%$ of 95 coronal-plane fragments were diagnosed with the use of conventional biplanar radiographs. The advantage of CT is that it can be used to assess intra-articular extension, metaphyseal comminution, and fracture lines in the coronal plane (9). The application of CT provides orthopaedic surgeons with a solid basis for further understanding of fracture morphology. Based on CT imaging, Xie et al. (10) previously described Hoffa fractures (Arbeitsgemeinschaft für Osteosynthesefragen Foundation/ Orthopaedic Trauma Association (AO/OTA) type 33B3.2 and type 33B3.3) morphologically and found quite a few fracture features imperative for optimal preoperative planning.

To the best of our knowledge, the AO/OTA fracture classification is the most widely used classification system for distal femur fractures. This classification system categorizes distal femur fractures into extra-articular (33A), partial articular (33B), and complete articular (33C) types (11). In this study, we focused on three AO/OTA types by describing the location and frequency of fracture lines of distal femur fractures through the 3-dimensional CT mapping technique $(12,13)$. The purpose of this study was to strengthen the understanding of morphological differences between three fracture types, hence providing more information for future biomechanical studies, operative treatment strategy, and new implant design. The level of evidence is level VI in this study. We present the following article in accordance with the STROBE reporting checklist (available at https://atm. amegroups.com/article/view/10.21037/atm-21-4591/rc).

\section{Methods}

\section{Participants}

Relying on the orthopaedic database of a large level-I trauma center, we conducted a retrospective search for the CT imaging data of patients diagnosed with a distal femur fracture between December 2016 and December 2020. Based on CT imaging data, the latest AO/OTA fracture classification (11) was used to categorize distal femur fractures. The 2-dimensional CT images and the 3 -dimensional reconstruction images in the picture archiving and communication system (PACS) were reviewed to identify the fracture classification. Once the fractures were confirmed by CT and categorized into AO/OTA fracture types 33A, 33B, or 33C, they were finally included in the analysis. Exclusion criteria included (I) patients $<18$ years of age; (II) patients with axial CT images with a slice thickness of $>3 \mathrm{~mm}$; (III) periprosthetic fractures around the knee joint; (IV) pathological fractures, and (V) developmental dysplasia. Finally, a total of 217 distal femur fractures in 216 patients were included. This retrospective study was performed in line with the principles of the Declaration of Helsinki (as revised in 2013). The study was approved by the Human Research Ethics Committee of Shanghai Sixth People's Hospital before the search of participants [No. 2016-129-(5)], and individual consent for this retrospective study was waived.

\section{Fracture mapping}

The 3-dimensional fracture mapping technique, which was previously applied in other fractures $(10,14)$, was adopted in this study (Figure 1). Two researchers (R Li and Y Zhuge) performed the fracture mapping under the supervision of an experienced orthopaedic surgeon (C Luo). Raw CT imaging data were obtained in the axial plane and saved as digital imaging and communications in medicine (DICOM) data. Then DICOM data were imported into Mimics software (version 17.0, Materialise NV Technologielaan, Leuven, Belgium) to be characterized simultaneously in the axial, 

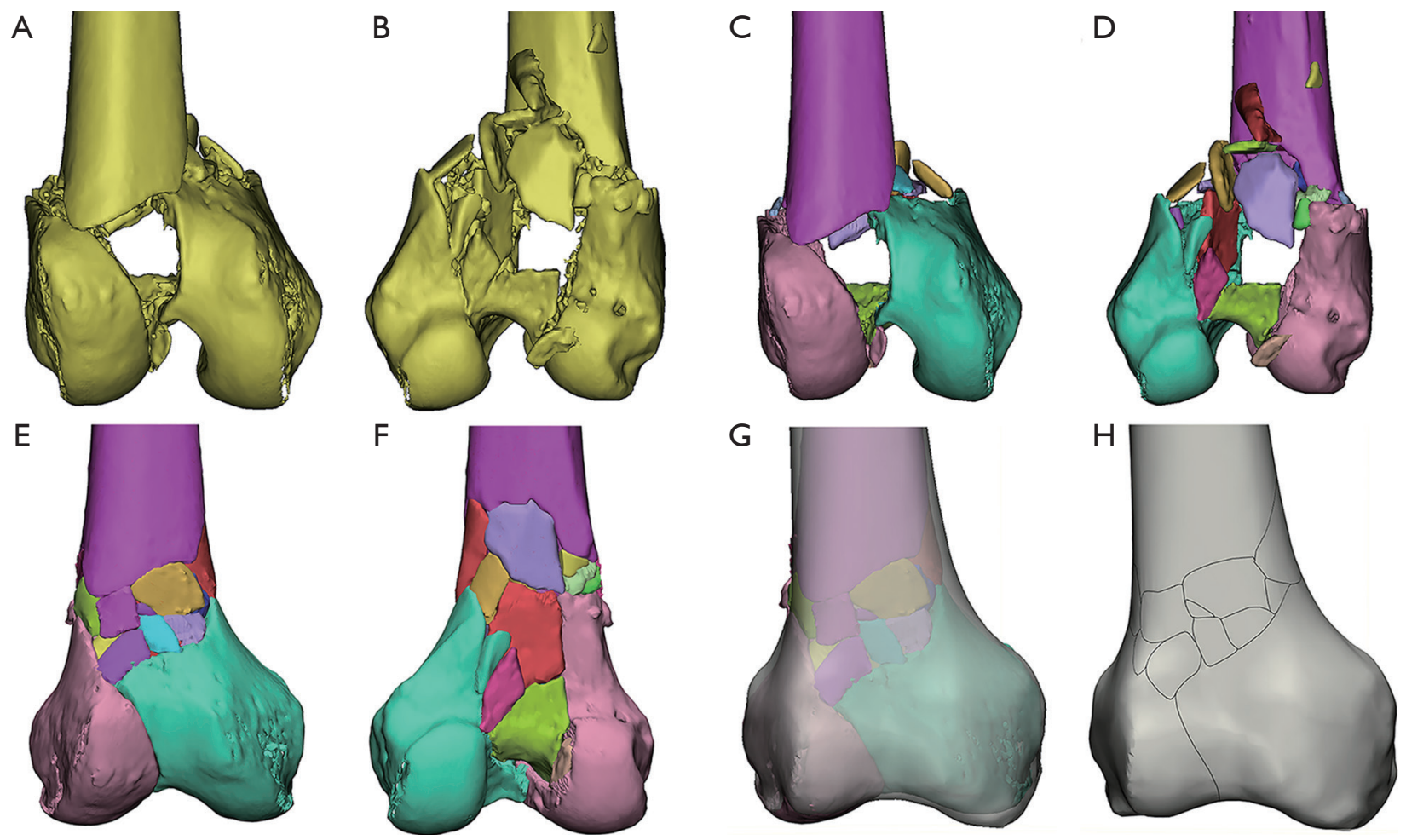

Figure 1 The flow diagram of the method used for 3-dimensional mapping of distal femur fractures. In this example of an Arbeitsgemeinschaft für Osteosynthesefragen Foundation/Orthopaedic Trauma Association (AO/OTA) fracture type C3 distal femur fracture, each fragment was reconstructed (A,B), segmented (C,D), and virtually reduced (E,F). Then fracture was mirrored (if left-sided) and fit onto the model of a distal third of the femur $(\mathrm{G})$. The osseous contours of the femur, especially the articular surface and the two condyles, were referenced for alignment and standardization. The contour of every fracture fragment was marked with smooth curves to delineate the fracture lines $(\mathrm{H})$.

sagittal, and coronal planes to prepare for the reconstruction of 3-dimensional fracture images. After the segmentation of fracture fragments, the distal femur fracture models were digitally reconstructed in Mimics software and virtually reduced. Data were then exported into 3-Matic software (version 12.0, Materialise NV Technologielaan, Leuven, Belgium) for 3-dimensional rendering and analyses. Rotation and scaling of the reconstructed fragments were performed when necessary to best match a 3-dimensional template model. The template was created using the CT imaging data of the right femur of a 30-year-old healthy man. The femur model was $495 \mathrm{~mm}$ in length, and the maximum width between the two condyles of the distal femur was $88 \mathrm{~mm}$. Since the reference template model was right-sided, all the left-sided fracture models were mirrored to match the model's orientation. The osseous contours of the distal third of the femur, especially the articular surface and the two condyles, were referenced for alignment and standardization. The contour of every fracture fragment was then marked with smooth curves directly on the surface of the template model to delineate the fracture lines. Finally, the overlap of all fracture lines allowed for the generation of 3-dimensional fracture maps. Heat maps based on the spatial frequency of fracture lines were created using E-3D software (15) (Central South University, Changsha, China; https://e3d-online.com), with blue to red indicating relatively low to high incidence.

\section{Statistical analysis}

Patient demographics and fracture characteristics are demonstrated as means and standard deviations (SDs) for continuous variables, and counts and percentages for categorical variables. A descriptive analysis of fracture maps was employed when the morphological features could not be presented quantitatively. For continuous variable 
Table 1 Patient demographics

\begin{tabular}{|c|c|}
\hline Demographic & Data $^{\dagger}$ \\
\hline \multicolumn{2}{|c|}{ Mean age (SD), years } \\
\hline Male & $51.1(13.7)^{\star}$ \\
\hline Female & $66.4(12.6)$ \\
\hline Total & $59.8(15.1)$ \\
\hline \multicolumn{2}{|l|}{ Sex, n (\%) } \\
\hline Male & $92(42.6)$ \\
\hline Female & $124(57.4)$ \\
\hline Total & $216(100.0)$ \\
\hline \multicolumn{2}{|l|}{ Knee, n (\%) } \\
\hline Left only & $114(52.8)$ \\
\hline Right only & $101(46.8)$ \\
\hline Bilateral & $1(0.4)$ \\
\hline Total & $216(100.0)$ \\
\hline \multicolumn{2}{|c|}{ AO/OTA classification, $n(\%)$} \\
\hline 33.A & $76(35.0)$ \\
\hline 33. $\mathrm{B}$ & $54(24.9)$ \\
\hline 33.C & $87(40.1)$ \\
\hline Total & $217(100.0)$ \\
\hline
\end{tabular}

${ }^{\dagger}$, data were presented as number (percentage), except for the patient age row as the mean (SD). The percentages shown were based on the total number of patients $(n=216)$ or fractures $(n=217) ;{ }^{*}, t$-test represented a significant difference between the age of male and female patients $(P<0.01)$. AO/ OTA, Arbeitsgemeinschaft für Osteosynthesefragen Foundation/ Orthopaedic Trauma Association; SD, standard deviation.

measures, between-group differences were analyzed using $t$-test and one-way analysis of variance (one-way ANOVA) after verifying that the data were normally distributed using the Shapiro-Wilk test. For categorical variables, they were analyzed using Chi-square tests. SPSS Statistics 20.0 software (IBM SPSS Inc., Armonk, NY, USA) was used for all the statistical analyses. $\mathrm{P}$ values less than 0.05 were considered statistically significant.

\section{Results}

\section{Patient demographics and fracture characteristics}

The patient demographics are summarized in Table 1. A total of 217 distal femur fractures in 216 patients were finally included. There were 114 (52.8\%) left knee injuries,
$101(46.8 \%)$ right knee injuries, and $1(0.4 \%)$ case with bilateral injury. The patient group comprised 92 (42.6\%) men and $124(57.4 \%)$ women ranging in age from 18 to 96 years (mean age 59.8 years, SD 15.1 years). The mean age of male patients was 51.1 years (SD 13.7 years) compared with 66.4 years (SD 12.6 years) in female patients, and there was a significant difference $(\mathrm{P}<0.01)$.

The frequency distribution of fractures grouped by patients' age-decade and sex is demonstrated in Figure $2 \mathrm{~A}$. When patients were $\leq 50$ years of age, distal femur fractures were more common in male patients in each decade. In contrast, more fractures were seen in female patients $>50$ years of age. Distal femur fractures were most likely to occur among patients aged 61 to 70 years in female patients, while patients aged 41 to 50 years and 61 to 70 years in male patients. All the three AO/OTA fracture types were most likely to occur among patients aged 61 to 70 years, as shown in Figure 2B. The fracture characteristics are summarized in Table 2. The mean age of patients with type A fractures was significantly higher than the other two types $(\mathrm{P}=0.013)$. In type $\mathrm{A}$ fractures, older patients and female patients were more common $(\mathrm{P}<0.01)$, while type $\mathrm{B}$ and type $\mathrm{C}$ fractures did not show this tendency. According to the AO/OTA classification (11), among the 217 distal femur fractures, there were 76 (35.0\%) type A fractures, 54 (24.9\%) type B fractures, and $87(40.1 \%)$ type $\mathrm{C}$ fractures (Figure 3). The distribution of AO/OTA fracture subtypes was as follows: 5 type A1, 13 type A2, 58 type A3, 14 type B1, 15 type B2, 25 type $\mathrm{B} 3,11$ type $\mathrm{C} 1,23$ type $\mathrm{C} 2$, and 53 type $\mathrm{C} 3$.

\section{Three-dimensional mapping}

All the 217 fracture lines were applied on a right-sided distal femur template (Figure 4). On the heat map, hot zones were mainly concentrated around the metaphysis, the lateral part of the intercondylar notch, and the patellofemoral joint. The densest frequency of fracture lines in the above three areas was 67 out of 217 .

Based on the surrounding view of the heat map (Figure 5), the fracture lines for AO/OTA type A fractures were mainly located around metaphysis, with the densest frequency of fracture lines in the anterior surface of the supracondylar area being 19 out of 76 . The fracture line hot zone on the medial column of the femoral metaphysis was more dispersed than on the lateral column. According to the scale of the template model, the distance of the medial hot zone was about $6 \mathrm{~cm}$, while the lateral side was about $4 \mathrm{~cm}$. A higher frequency of fracture line density demonstrated that 

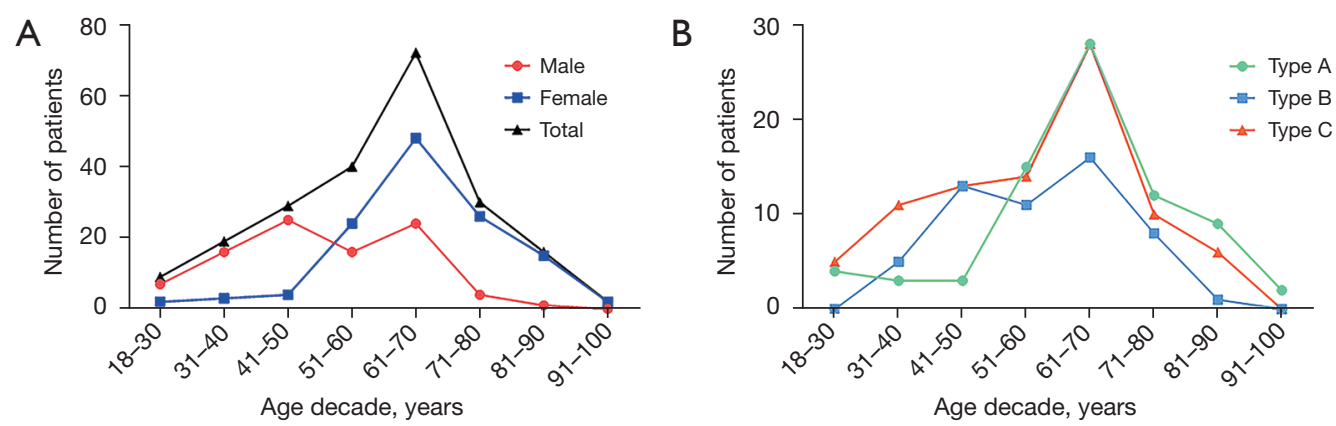

Figure 2 The frequency distribution of distal femur fractures. (A) Distribution grouped by patients' age-decade and sex. (B) Distribution grouped by patients' age-decade and Arbeitsgemeinschaft für Osteosynthesefragen Foundation/Orthopaedic Trauma Association (AO/OTA) fracture type.

Table 2 Fracture characteristics

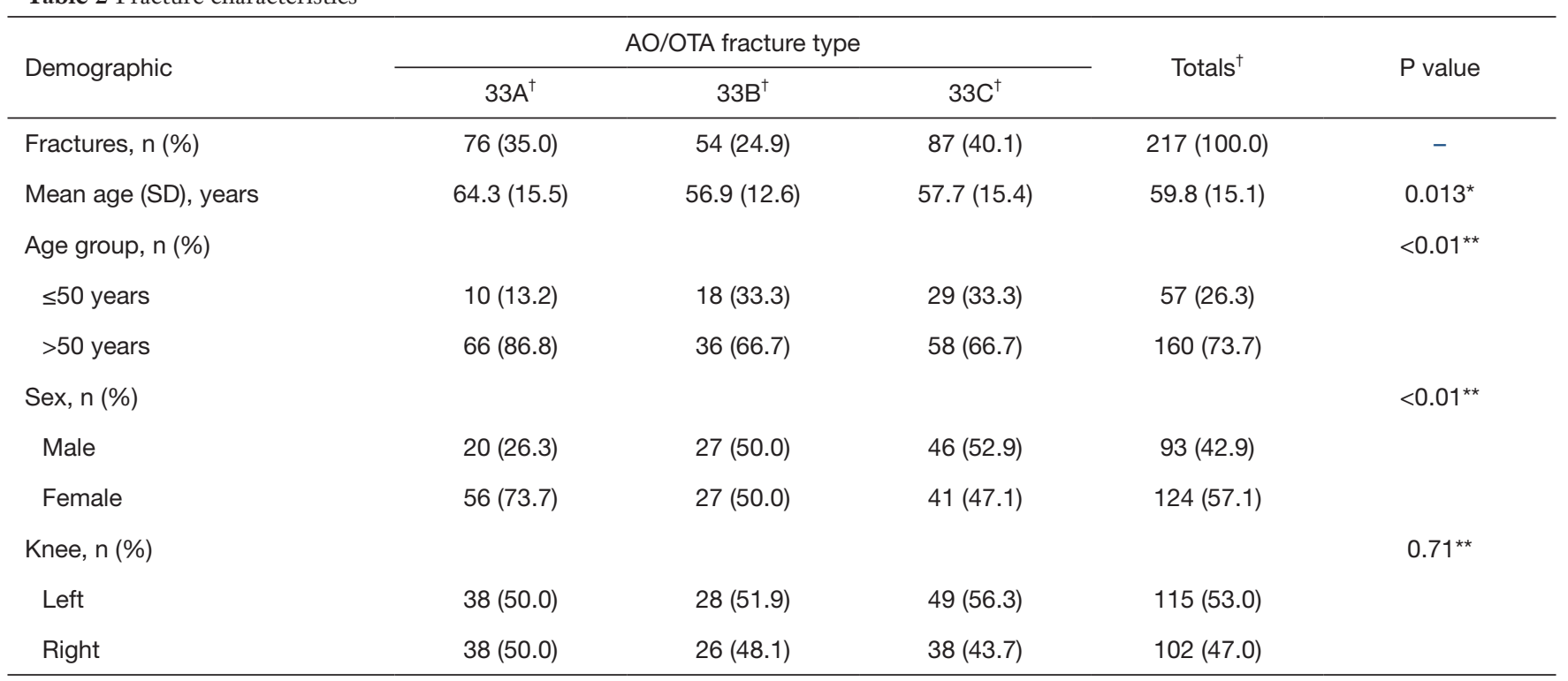

${ }^{\dagger}$, data were presented as number (percentage), except for the patient age row as the mean (SD). The percentages shown were based on the number of fractures for each fracture type or the total number $(n=217)$; *, significant differences evaluated by one-way analysis of variance (ANOVA), there were significant differences between group 33A and the other three groups; ${ }^{* *}$, Chi-square tests for age group, sex, and side of injury among the three fracture types. AO/OTA, Arbeitsgemeinschaft für Osteosynthesefragen Foundation/Orthopaedic Trauma Association; SD, standard deviation.

fracture lines mainly extended from the superomedial to the inferolateral part of the supracondylar area. The peak point of the fracture line compact area was approximately $10 \mathrm{~cm}$ proximal to the joint line on the medial side, and the lowest point was $4 \mathrm{~cm}$ on the lateral side.

In the 54 type B fractures, fracture lines were mostly concentrated around the intercondylar notch, with the hot zone located slightly laterally (Figure 6). The densest frequency of fracture lines in the articular surface was 30 out of 54. From the posterior view, fracture lines extended posteriorly to the position near the anterior cruciate ligament (ACL) footprint.

In the 87 type $\mathrm{C}$ fractures, fracture lines were mostly concentrated around the lateral part of the intercondylar notch and the metaphysis (Figure 7). On the heat map, the hot zone demonstrated a "Y" sign. Specifically, the hot zone of the intercondylar notch constituted the lower half of the "Y". The hot zone of the metaphysis extended from the patellofemoral joint and then obliquely upwards to the lateral and medial sides of the metaphysis, which constituted 


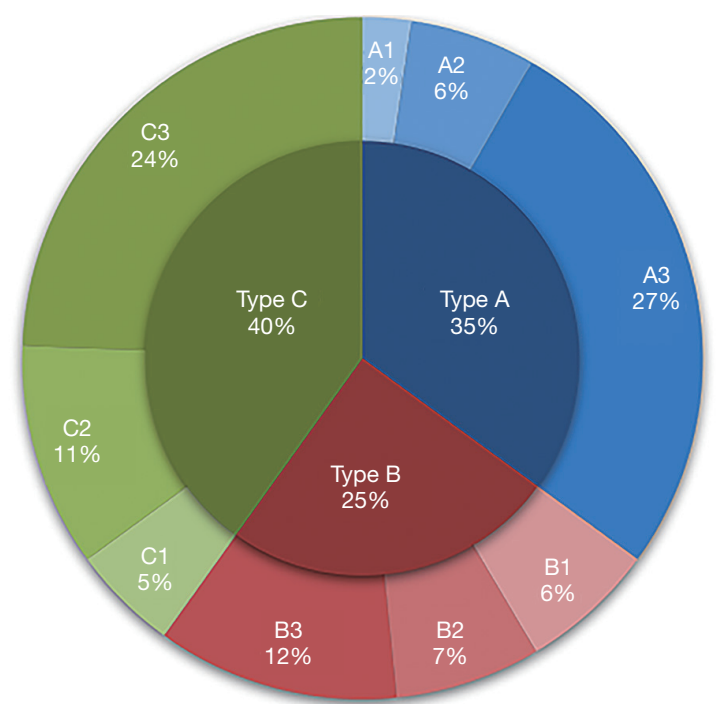

Figure 3 The percentages of three Arbeitsgemeinschaft für Osteosynthesefragen Foundation/Orthopaedic Trauma Association (AO/OTA) fracture types and subtypes in 217 distal femur fractures.
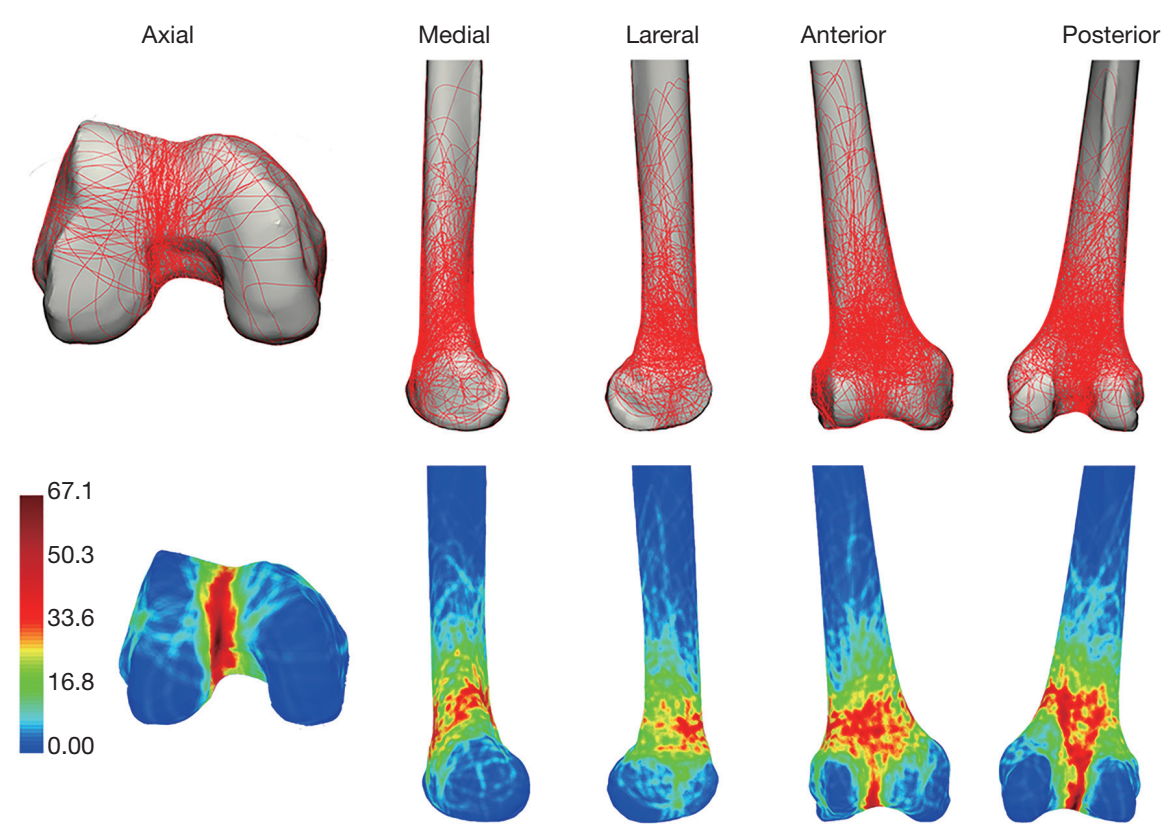

Figure 4 The upper section shows representative views of the 3-dimensional distal femur template with all Arbeitsgemeinschaft für Osteosynthesefragen Foundation/Orthopaedic Trauma Association (AO/OTA) type A, B, and C fracture lines (n=217). The lower section demonstrates the heat map, including the axial, medial, lateral, anterior, and posterior views. Red color represents a higher fracture line density.

the upper half of the "Y". The distribution of fracture lines was consistent with the type C classification system (11). The densest frequency of fracture lines located in the lateral border of the articular surface was 38 out of 87 . From the surrounding view, the medial and lateral concentration areas of fracture lines in the metaphysis were at the same height level proximal to the joint line. Based on the scale of the bone template, the distance from the lower border 


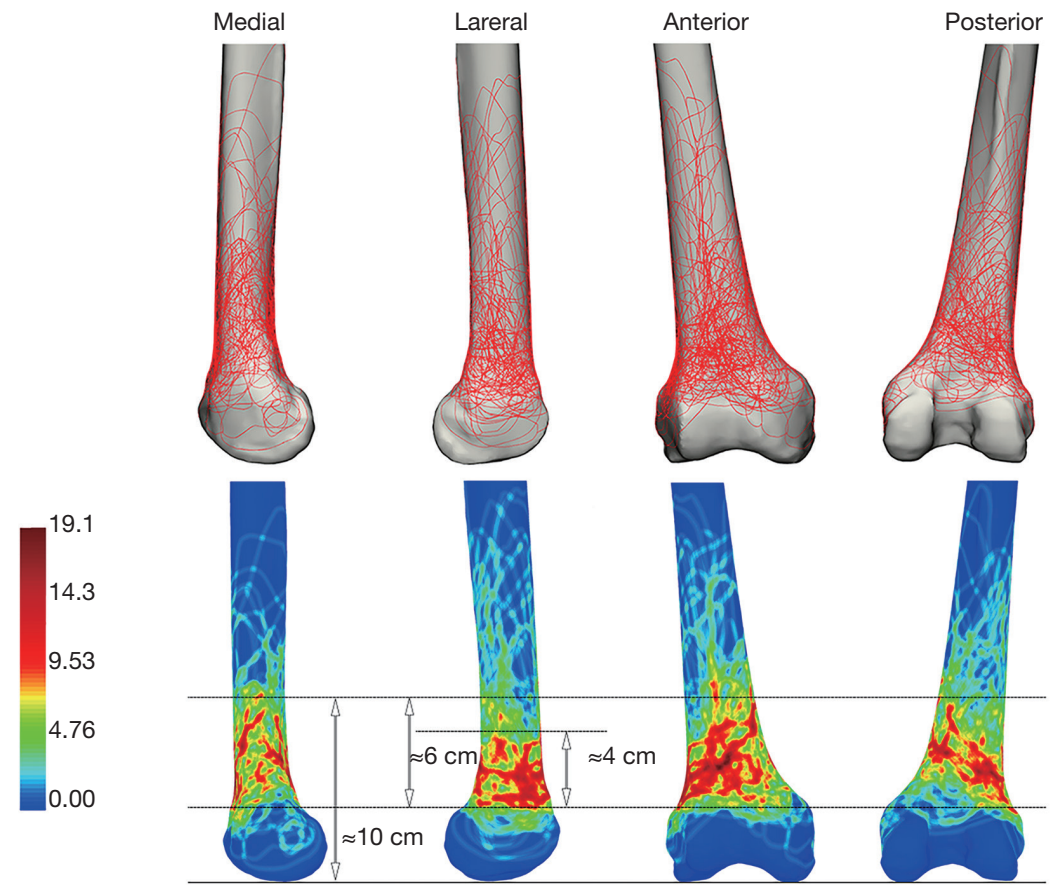

Figure 5 The upper section shows representative views of the 3-dimensional distal femur template with Arbeitsgemeinschaft für Osteosynthesefragen Foundation/Orthopaedic Trauma Association (AO/OTA) type A fracture lines (n=76). The lower section demonstrates the heat map, including the medial, lateral, anterior, and posterior views. Red color represents a higher fracture line density. On the template model, the distance of the medial hot zone was about $6 \mathrm{~cm}$, while the lateral side was $4 \mathrm{~cm}$. The peak point of the fracture line compact area was approximately $10 \mathrm{~cm}$ proximal to the joint line on the medial side, and the lowest point was $4 \mathrm{~cm}$ on the lateral side.
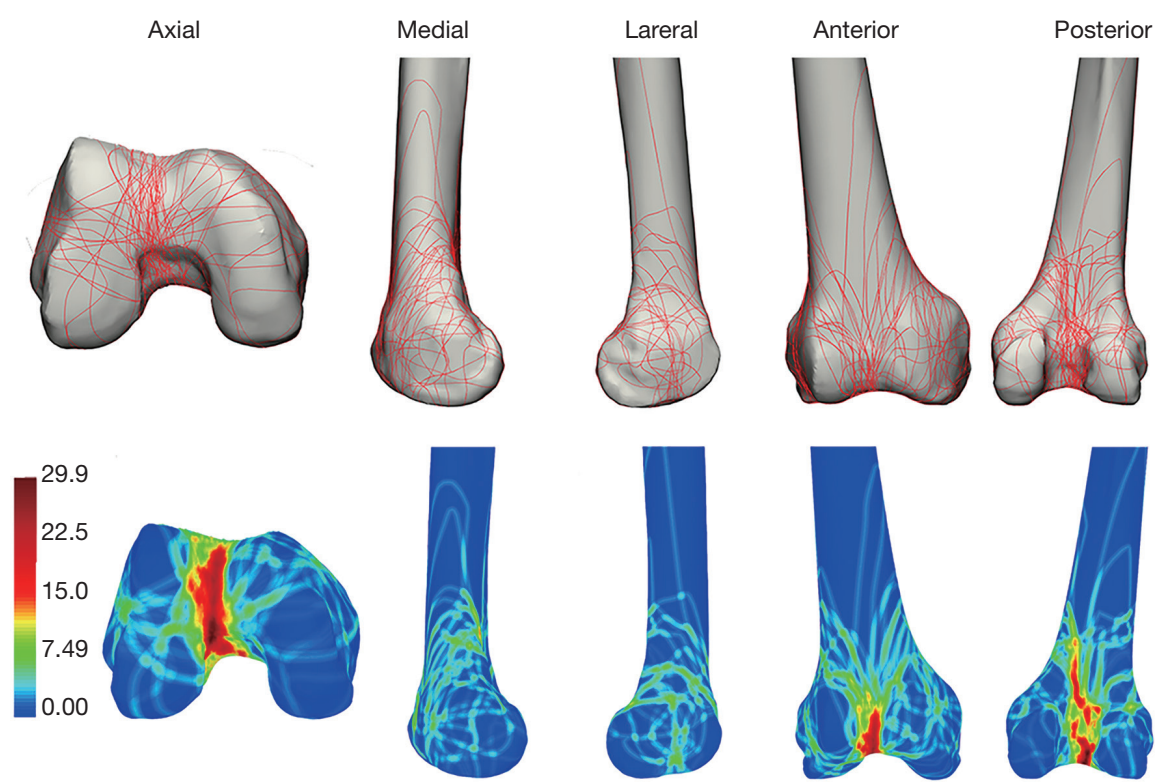

Figure 6 The upper section shows representative views of the 3-dimensional distal femur template with Arbeitsgemeinschaft für Osteosynthesefragen Foundation/Orthopaedic Trauma Association (AO/OTA) type B fracture lines ( $\mathrm{n}=54$ ). The lower section demonstrates the heat map, including the axial, medial, lateral, anterior, and posterior views. Red color represents a higher fracture line density. 

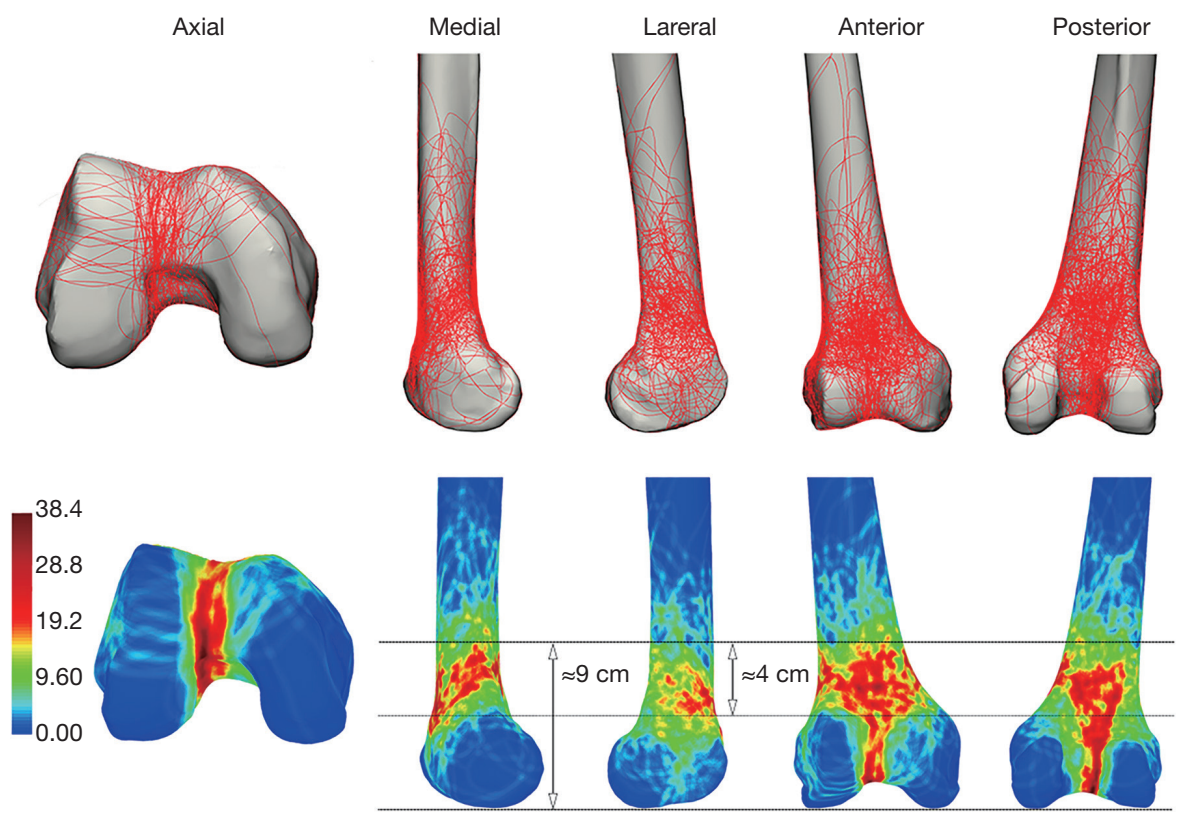

Figure 7 The upper section shows representative views of the 3-dimensional distal femur template with Arbeitsgemeinschaft für Osteosynthesefragen Foundation/Orthopaedic Trauma Association (AO/OTA) type $\mathrm{C}$ fracture lines ( $\mathrm{n}=87$ ). The lower section demonstrates the heat map, including the axial, medial, lateral, anterior, and posterior views. Red color represents a higher fracture line density. On the template model, the distance from the lower border of the hot zone to the joint line was approximately $5 \mathrm{~cm}$, and the upper end was $9 \mathrm{~cm}$. The perpendicular distance of the hot zone was about $4 \mathrm{~cm}$.

of the hot zone to the joint line was approximately $5 \mathrm{~cm}$, and the upper end was $9 \mathrm{~cm}$. Therefore, the perpendicular distance of the supracondylar higher frequency of fracture line density was about $4 \mathrm{~cm}$. Only the posterolateral part of the metaphysis was less concentrated with fracture lines than other areas of the metaphysis.

For intercondylar fractures, there were 26 (48.1\%) coronal plane fractures in 54 type $\mathrm{B}$ fractures and 32 (36.8\%) in 87 type $\mathrm{C}$ fractures. In total, there were 58 fractures $(41.1 \%)$ in 141 intercondylar fractures. Based on the 3-dimensional maps, the lateral condyle was found to be more frequently involved $(33 / 58,56.9 \%)$.

\section{Discussion}

In this retrospective study, the 3-dimensional fracture mapping technique was applied to 217 distal femur fractures. Compared with the 2 -dimensional fracture mapping technique, the 3-dimensional mapping can delineate fracture morphology more precisely (16). Based on these fracture maps and heat maps, some distinct characteristics of distal femur fractures were found. The fracture pattern of the articular surface in cases of AO/OTA type $\mathrm{B}$ and type $\mathrm{C}$ fractures shared the same features. There was a concentration of fracture lines in the lateral part of the intercondylar notch, and the hot zones extended posteriorly to the area near the ACL footprint (17), which suggests that special attention should be paid to concomitant ligamentous injury of the distal femur fractures during the preoperative physical examination and intraoperative exploration. However, the fracture features in the supracondylar part of AO/OTA type A and type C fractures differed. In type A fractures, the higher frequency of fracture lines was located from superomedial to inferolateral. In contrast, in type $\mathrm{C}$ fractures, the supracondylar fracture lines were almost located on the same height level just above the femoral condyles.

The findings of fracture features can aid in designing the fracture model in biomechanical studies. Currently, there is no consistent osteotomy protocol for the creation of a distal femur fracture model, especially for type A and type $\mathrm{C}$ fractures with comminuted metaphysis. To create a type A3 fracture model, Park et al. (18) made a $2.5-\mathrm{cm}$ osteotomy gap at $6 \mathrm{~cm}$ proximal from the distal articular surface, while Wright et al. (19) made a $2-\mathrm{cm}$ gap $6.5 \mathrm{~cm}$ proximal to the intercondylar notch. For type $\mathrm{C}$ fractures, 
Zhang et al. (20) produced a $1.5-\mathrm{cm}$ osteotomy plane at a point $6.5 \mathrm{~cm}$ proximal to the joint line to create a femur model of type C2 fracture. Duffy et al. (21) created a supracondylar $1-\mathrm{cm}$ fracture gap $6 \mathrm{~cm}$ proximal to the joint line to simulate a C3 fracture. Meanwhile, Fontenot et al. (22) simulated a type $\mathrm{C}$ fracture by cutting a $1-\mathrm{cm}$ osteotomy gap located $5 \mathrm{~cm}$ proximal to the most distal surface of the trochlear groove and medial comminution with an additional $3-\mathrm{cm}$ angled medial wedge. All the bone models used for biomechanical tests in the above studies were composite models instead of cadaveric femurs. For the intercondylar osteotomy plane of the fracture model in these studies (20-22), a sagittal cut was made directly between both condyles through the intercondylar notch. Our study found the features of AO/OTA type A and type $\mathrm{C}$ fractures on the heat maps. Since the cases of type A and type $\mathrm{C}$ fractures were mostly comprised of fractures with comminuted metaphysis (types A3, C2, and C3) (Figure 3), the study results can be helpful for creating the osteotomy gap. Based on the scale of the bone template, the length of the medial hot zone was measured about $6 \mathrm{~cm}$, while the lateral side was about $4 \mathrm{~cm}$ in type $A$ fractures. The upper end of the area was approximately $10 \mathrm{~cm}$ proximal to the joint line on the medial side, and the lowest point was $4 \mathrm{~cm}$ on the lateral side. In type $\mathrm{C}$ fractures, the distance from the lower border of the fracture line hot zone to the joint line was approximately $5 \mathrm{~cm}$, and the perpendicular length of the hot zone was about $4 \mathrm{~cm}$. Although the distance of the hot zone measured on the femur template can only be used as a reference, osteotomy gaps created in the former studies (18-22) were not long enough to accurately simulate the fracture morphology, which may underpower the results drawn from those studies. According to the scale of the template model, we suggest that for type A fracture model, the osteotomy gap should be made approximately $4 \mathrm{~cm}$ and the supracondylar osteotomy at $4 \mathrm{~cm}$ proximal to the joint line. For type $\mathrm{C}$ fracture model, the osteotomy gap should be made $4 \mathrm{~cm}$ and the supracondylar osteotomy at $5 \mathrm{~cm}$ proximal to the joint line. For the sagittal intercondylar osteotomy plane, it should be produced more laterally in the intercondylar notch. Thus, biomechanical studies adopting fracture models based on our findings may draw conclusions different from previous studies.

Improved understanding of distal femur fracture morphology is essential for preoperative planning and implant fixation strategy. For distal femur fractures with comminuted metaphysis, such as types 33A3, 33C2, and $33 \mathrm{C} 3$, delayed union or nonunion, hardware failure, and loss of alignment after the operation remain challenging problems (4-7). According to the heat maps in this study, the supracondylar part of type $\mathrm{C}$ fractures was demonstrated as bi-columnar involvement, which suggests that additional medial column support should be considered to achieve a stable fixation in some cases. Recently, dual plating has been used to treat some complex distal femur fractures with severe comminution or osteopenia. Studies demonstrated that adding a medial plate after lateral plating led to good radiological and clinical results $(6,23)$. The biomechanical study proved that the medial plate opposed varus stress of the lower limb, shared part of stresses from the lateral plating (20), and thus increased the fracture stability (18). Rollick et al. (24) reported that an addition of a plate to the medial side of the distal femur after a laterally based plate did not further influence the vascularization of the femur. Besides the lateral surgical approach, additional medial exposure may be needed for severely comminuted supracondylar fractures in which the medial plate is required. In type $\mathrm{C}$ fractures, an addition of a medial subvastus approach is needed for the medial support since the fracture lines were concentrated around the metaphysis. For type A fractures in this study, the supracondylar fracture pattern presented as an oblique shape with fracture lines more dispersed on the medial column of the metaphysis. For this reason, a medial subvastus approach may not be able to fully expose the medial side of type A3 fractures if double plating is used. Therefore, a modified surgical incision to the distal femur such as the "Swashbuckler" approach described by Starr et al. (25) should be considered by orthopaedic surgeons in preoperative planning. Meanwhile, although the biomechanical study has found that double plate constructs provided more stable fixation than lateral plate + retrograde intramedullary nail (rIMN) constructs (19), lateral plate + rIMN constructs may be more suitable in type A3 fracture cases with dispersed medial comminuted metaphysis due to a lack of medial exposure.

The presence of coronal plane fractures in an intraarticular distal femur fracture has received more attention in recent years. In this study, there were 58 coronal plane fractures $(41.1 \%)$ presented in 141 intercondylar fractures, which was a rate consistent with previous research $(8,26)$. This suggests that coronal fracture fragments account for a considerable proportion of intra-articular distal femur fractures; therefore, orthopaedic surgeons should pay special attention to coronal plane fracture fragments during the management of the AO/OTA type $\mathrm{B}$ and type $\mathrm{C}$ distal femur fractures. 
There were some limitations in this study. First, this study only involved the morphological description of fractures without further research on related complications, fracture surgery, and patient prognosis. Second, since the reconstruction of distal femur fractures and morphologic analyses were based on CT imaging, the final results may not have been representative of those fractures with no CT available. Moreover, fracture lines depicted were only limited on the distal femur surface, and the comminution zone was not further explored in this study. Therefore, some fracture characteristics, such as fragment impaction, were not evaluated. Finally, since all the data was from a single-centered database, only patients in a regional area were included in this study. Different regions, ethnicity, and social development might be potential influence factors (27) and should be taken into consideration in future studies. Nonetheless, this is the first study that systematically described the fracture characteristics of a large number of distal femur fractures $(n=217)$.

\section{Conclusions}

This study elucidated the detailed morphology of distal femur fractures through the analysis of a series of 3-dimensional fracture maps. In conclusion, three AO/OTA fracture types had distinct characteristics. The intercondylar fracture patterns in AO/OTA type $\mathrm{B}$ and type $\mathrm{C}$ fractures were similar, while the supracondylar characteristics in $\mathrm{AO} /$ OTA type A and type $\mathrm{C}$ were different. These findings obtained from the maps can help orthopaedic surgeons better understand the fracture morphology. Further studies are needed to establish a standard biomechanical fracture model and optimize fixation strategy.

\section{Acknowledgments}

The authors wish to thank Dr. Yingqi Zhang for providing technical support for fracture line heat maps.

Funding: This work was supported by AO Foundation, AO Innovation Translation Center (No. LEC12). AO Innovation Translation Center is an organizational unit of the AO Foundation, which is an independent medically guided not-for-profit organization based in Switzerland.

\section{Footnote}

Reporting Checklist: The authors have completed the STROBE reporting checklist. Available at https://atm. amegroups.com/article/view/10.21037/atm-21-4591/rc

Data Sharing Statement: Available at https://atm.amegroups. com/article/view/10.21037/atm-21-4591/dss

Conflicts of Interest: All authors have completed the ICMJE uniform disclosure form (available at https://atm. amegroups.com/article/view/10.21037/atm-21-4591/coif). The authors report that this study was granted by AO Innovation Translation Center of the AO Foundation to reveal the morphologic details of the distal femur fractures (AO/OTA types 33A, 33B, and 33C). The authors have no other conflicts of interest to declare.

Ethical Statement: The authors are accountable for all aspects of the work in ensuring that questions related to the accuracy or integrity of any part of the work are appropriately investigated and resolved. This retrospective study was performed in line with the principles of the Declaration of Helsinki (as revised in 2013). The study was approved by the Human Research Ethics Committee of Shanghai Sixth People's Hospital before the search of participants [No. 2016-129-(5)], and individual consent for this retrospective study was waived.

Open Access Statement: This is an Open Access article distributed in accordance with the Creative Commons Attribution-NonCommercial-NoDerivs 4.0 International License (CC BY-NC-ND 4.0), which permits the noncommercial replication and distribution of the article with the strict proviso that no changes or edits are made and the original work is properly cited (including links to both the formal publication through the relevant DOI and the license). See: https://creativecommons.org/licenses/by-nc-nd/4.0/.

\section{References}

1. Court-Brown CM. The epidemiology of fractures and dislocations. In: Court-Brown CM. editor. Rockwood and Green's fractures in adults. Philadelphia: Walters Kluwer, 2015:63.

2. Martinet $\mathrm{O}$, Cordey J, Harder $\mathrm{Y}$, et al. The epidemiology of fractures of the distal femur. Injury 2000;31 Suppl 3:C62-3.

3. Elsoe R, Ceccotti AA, Larsen P. Population-based epidemiology and incidence of distal femur fractures. Int Orthop 2018;42:191-6.

4. Henderson CE, Lujan TJ, Kuhl LL, et al. 2010 mid- 
America Orthopaedic Association Physician in Training Award: healing complications are common after locked plating for distal femur fractures. Clin Orthop Relat Res 2011;469:1757-65.

5. Kim JW, Oh CW, Oh JK, et al. Malalignment after minimally invasive plate osteosynthesis in distal femoral fractures. Injury 2017;48:751-7.

6. Bologna MG, Claudio MG, Shields KJ, et al. Dual plate fixation results in improved union rates in comminuted distal femur fractures compared to single plate fixation. J Orthop 2020;18:76-9.

7. Henderson CE, Kuhl LL, Fitzpatrick DC, et al. Locking plates for distal femur fractures: is there a problem with fracture healing? J Orthop Trauma 2011;25 Suppl 1:S8-14.

8. Nork SE, Segina DN, Aflatoon K, et al. The association between supracondylar-intercondylar distal femoral fractures and coronal plane fractures. J Bone Joint Surg Am 2005;87:564-9.

9. Gwathmey FW Jr, Jones-Quaidoo SM, Kahler D, et al. Distal femoral fractures: current concepts. J Am Acad Orthop Surg 2010;18:597-607.

10. Xie X, Zhan Y, Dong M, et al. Two and ThreeDimensional CT Mapping of Hoffa Fractures. J Bone Joint Surg Am 2017;99:1866-74.

11. Meinberg EG, Agel J, Roberts CS, et al. Fracture and Dislocation Classification Compendium-2018 J Orthop Trauma 2018;32 Suppl 1:S1-S170.

12. Cole PA, Mehrle RK, Bhandari M, et al. The pilon map: fracture lines and comminution zones in OTA/AO type 43C3 pilon fractures. J Orthop Trauma 2013;27:e152-6.

13. Armitage BM, Wijdicks CA, Tarkin IS, et al. Mapping of scapular fractures with three-dimensional computed tomography. J Bone Joint Surg Am 2009;91:2222-8.

14. Xie X, Zhan Y, Wang Y, et al. Comparative Analysis of Mechanism-Associated 3-Dimensional Tibial Plateau Fracture Patterns. J Bone Joint Surg Am 2020;102:410-8.

15. Zhang Y, Sun Y, Liao S, et al. Three-Dimensional Mapping of Medial Wall in Unstable Pertrochanteric Fractures. Biomed Res Int 2020;2020:8428407.

16. Dugarte AJ, Tkany L, Schroder LK, et al. Comparison of 2 versus 3 dimensional fracture mapping strategies for 3

Cite this article as: Li R, Zhuge Y, Zhan Y, Xie X, Luo C. Three-dimensional computed tomography mapping and analysis of distal femur fractures (AO/OTA types 33A, 33B, and 33C). Ann Transl Med 2022;10(7):398. doi: 10.21037/atm-214591 dimensional computerized tomography reconstructions of scapula neck and body fractures. J Orthop Res 2018;36:265-71.

17. Ferretti M, Ekdahl M, Shen W, et al. Osseous landmarks of the femoral attachment of the anterior cruciate ligament: an anatomic study. Arthroscopy 2007;23:1218-25.

18. Park KH, Oh CW, Park IH, et al. Additional fixation of medial plate over the unstable lateral locked plating of distal femur fractures: A biomechanical study. Injury 2019;50:1593-8.

19. Wright DJ, DeSanto DJ, McGarry MH, et al. Supplemental Fixation of Supracondylar Distal Femur Fractures: A Biomechanical Comparison of Dual-Plate and Plate-Nail Constructs. J Orthop Trauma 2020;34:434-40.

20. Zhang W, Li J, Zhang H, et al. Biomechanical assessment of single LISS versus double-plate osteosynthesis in the AO type 33-C2 fractures: A finite element analysis. Injury 2018;49:2142-6.

21. Duffy P, Trask K, Hennigar A, et al. Assessment of fragment micromotion in distal femur fracture fixation with RSA. Clin Orthop Relat Res 2006;448:105-13.

22. Fontenot PB, Diaz M, Stoops K, et al. Supplementation of Lateral Locked Plating for Distal Femur Fractures: A Biomechanical Study. J Orthop Trauma 2019;33:642-8.

23. Steinberg EL, Elis J, Steinberg Y, et al. A double-plating approach to distal femur fracture: A clinical study. Injury 2017;48:2260-5.

24. Rollick NC, Gadinsky NE, Klinger CE, et al. The effects of dual plating on the vascularity of the distal femur. Bone Joint J 2020;102-B:530-8.

25. Starr AJ, Jones AL, Reinert CM. The "swashbuckler": a modified anterior approach for fractures of the distal femur. J Orthop Trauma 1999;13:138-40.

26. Richards JA, Berkay FB, Davis CM, et al. Intra-articular Fracture Pattern in Intercondylar Distal Femur Fractures: An Analysis of Frequency and Major Fracture Fragments. Injury 2021;52:967-70.

27. Everhart JS, Chaudhari AM, Flanigan DC. Creation of a simple distal femur morphology classification system. J Orthop Res 2016;34:924-31. 